\title{
ON AUTOMATIC SURJECTIVITY OF SPECTRUM PRESERVING ADDITIVE TRANSFORMATIONS
}

\author{
El Houcine El Bouchibti \\ Polydisciplinary Faculty - Taroudant \\ Ibno Zohr university \\ B.P. 271, CP 83000, Hay Lastah Taroudant, MOROCCO
}

\begin{abstract}
Let $X$ and $Y$ be an infinite dimensional complex Banach spaces and let $\Phi$ : $B(X) \longrightarrow B(Y)$ be a spectrum preserving additive transformation. We show that if the range of $\Phi$ contains the ideal of finite rank operators of $B(Y)$, then either $\Phi(T)=A T A^{-1}$ or $\Phi(T)=B T^{*} B^{-1}$ for every $T \in B(X)$, where $A: X \longrightarrow Y$ and $B: X^{\prime} \longrightarrow Y$ are linear bounded isomorphisms.
\end{abstract}

AMS Subject Classification: 15A04, 15A86, 47B48, 47B49

Key Words: linear preserver problem, trace, operator algebra, analytic functions

\section{Introduction}

Throughout this paper we will denote by $X$ and $Y$ infinite dimensional complex Banach spaces, $X^{\prime}$ the dual of $X, B(X)$ and $B(Y)$ the algebras of all linear bounded operators on $X$ and $Y$ respectively. It seems that in the last few years there has been growing interest in problems of characterizing transformations preserving some sets between the algebras of linear bounded operators over infinite dimensional Banach spaces, we can see for example [1], [2], [3], [4], [9], [11], [12], [13], [15], [16], [18]. It has been proved by B.Aupetit [7] that if

$\begin{array}{lr}\text { Received: } & \text { April 29, } 2016 \\ \text { Revised: } & \text { November 15, 2016 } \\ \text { Published: } & \text { February 7, 2017 }\end{array}$

(C) 2017 Academic Publications, Ltd. url: www.acadpubl.eu 
$\Phi: B(X) \longrightarrow B(Y)$ is a spectrum preserving surjective additive transformation, then either $\Phi(T)=A T A^{-1}$ or $\Phi(T)=B T^{*} B^{-1}$ for every $T \in B(X)$, where $A: X \longrightarrow Y$ and $B: X^{\prime} \longrightarrow Y$ are linear bounded isomorphisms. He asked the question what happens if such transformation is not surjective. In our paper [3] we have get the same result of B.Aupetit [7] by the same hypotheses but we assumed that only the image of quasi-nilpotent operators contains all quasi nilpotent operators. In this paper we propose to have the same results of B.Aupetit by the same hypotheses but we assume only that the range of $\Phi$ contains all finite rank operators.

Let us fix some notations. For every $T \in B(X)$ we denote by $\sigma(T)$ and $\rho(T)$ the spectrum and the spectral radius of $T$ respectively. Every operator on $X$ of rank one can be written as $x \otimes f$ for some $x \in X-\{0\}$ and $f \in X^{\prime}-\{0\}$. We recall that $x \otimes f$ is defined by $(x \otimes f) y=f(y) x$ for $y \in X$. The operator $x \otimes f$ is idempotent if and only if $f(x)=1$, moreover $\sigma(x \otimes f)=\{0, f(x)\}$ ( see[12] and [13]). We denote by $F(X)$ the ideal of finite rank operators it coincides with $\operatorname{Soc} B(X)$, the socle of $B(X)$. We know by [6] that the socle of $B(X)$ is linearly engendered by idempotent operators of rank one. $A n n \operatorname{Soc} B(X)$ denotes the set of operators $T \in B(X)$ satisfying $T R=0$ for every $R \in \operatorname{Soc} B(X)$. $\operatorname{Im} \Phi$ denotes the range of $\Phi$. We denote by $\lambda$ the operator $\lambda . I$ for every $\lambda \in \mathbb{C}$ and by $F_{1}(X)$ the set of rank one operators of $B(X)$.

\section{Main Results}

The aim of this work is to prove the following results:

Theorem 2.1. Let $\Phi: B(X) \longrightarrow B(Y)$ be a spectrum preserving additive transformation such that $F(Y) \subset \operatorname{Im} \Phi$. Then either:

i) $\Phi(T)=A T A^{-1}$, for every $T \in B(X)$, where $A: X \longrightarrow Y$ is a bounded bijective transformation; or

ii) $\Phi(T)=B T^{*} B^{-1}$ for every $T \in B(X)$, where $B: X^{\prime} \longrightarrow Y$ is a bounded bijective linear transformation.

Corollary 2.1. Let $\Phi: B(X) \longrightarrow B(X)$ be a spectrum preserving additive transformation such that $F(X) \subset \operatorname{Im} \Phi$. Then $\Phi$ is surjective.

In order to prove those results we are going to use the notion of trace, which generalise the trace of matrices. in [6] B.Aupetit defines the trace of an operator $T \in F(X)$ by

$$
\operatorname{Tr}(T)=\sum_{\lambda \in \sigma(a)} \lambda m(\lambda, T),
$$


where for every $\lambda \in \sigma(T), m(\lambda, T)$ denotes the multiplicity of $\lambda$ in the spectrum of $T$. In fact B.Aupetit shows in [6] that this multiplicity is equal to the rank of the Riesz projection associated to $T$ and $\alpha$. Let $\alpha \in \sigma(T)$ and $\Gamma$ be a small curve isolating $\alpha$ from the rest of the spectrum of $T$. By definition the Riesz projection is

$$
P(\alpha, T)=\frac{1}{2 \pi i} \int_{\Gamma}(\lambda e-T)^{-1} d \lambda .
$$

This multiplicity coincides with the multiplicity of eigenvalues in the case of matrices. The trace has the following properties(see [6]):

\section{Properties:}

i) $\operatorname{Tr}(S+T)=\operatorname{Tr}(S)+\operatorname{Tr}(T)$ for every $S, T \in F(X)$.

ii) $\sigma(R)=\{0, \operatorname{Tr}(R)\}$ for every $R \in F_{1}(X)$.

iii) $\operatorname{Tr}(\alpha T)=\alpha \operatorname{Tr}(T)$ for every $\alpha \in \mathbb{C}$.

iv) The function $T \longrightarrow \operatorname{Tr}(T)$ is continuous over $F_{n}(X)$, for every $n \in \mathbb{N}^{*}$.

v) Let $f$ be an analytic function from a domain $D$ of $\mathbb{C}$ into $F(X)$. Then the function $\lambda \longrightarrow \operatorname{Tr}(f(\lambda))$ is holomorphic on $D$.

The following lemma is a simple version of result of B.Aupetit in the case of $B(X)$ (see[6] Corollary 3.6).

Lemma 2.1. Let $S \in B(X)$. If $\operatorname{Tr}(S T)=0$ for every $T \in B(X)$. Then $S=0$.

Proof. It suffices to see that $\operatorname{AnnSocB}(X)=\{0\}([6]$ and [7]).

The following lemma is due to B.Aupetit [7] Corollary 2.4.

Lemma 2.2. let $\Phi: B(X) \longrightarrow B(Y)$ be a spectrum preserving additive transformation. Then $\operatorname{rank}(T) \leq \operatorname{rank} \Phi(T)$ for every $T \in B(X)$. In particular $\Phi$ is injective.

This lemma is an amelioration of one result of P. ̌́ emrl, see [12]

Lemma 2.3. Let $R$ be non zero operator in $B(X)$. Then the following conditions are equivalent:

i) $\operatorname{rank} R=1$,

ii) $\sigma(T+R) \cap \sigma(T+2 R) \subset \sigma(T)$, for every $T \in F(X)$.

Proof. Let us prove that i) implies ii). Let $R \in B(X)$ be an operator of rank one. Suppose there exists $T \in F(X)$ such that $\sigma(T+R) \cap \sigma(T+2 R) \not \subset \sigma(T)$. Then there exists $\lambda \in \mathbb{C}$ such that $\lambda-T$ is invertible, and $\lambda-T-R$ and $\lambda-T-2 R$ are not invertible operators. Therefore $I-(\lambda-T)^{-1} R$ and $I-2(\lambda-2 T)^{-1} R$ 
are not invertible operators. Thus $\frac{1}{2} \in \sigma\left((\lambda I-T)^{-1} R\right)$ which contradicts the fact $(\lambda-T)^{-1} R$ is of rank one.

We prove now that ii) implies i). Assume that ii) is satisfied and $\operatorname{rank} R>$ 1. Suppose that there exists $x \in X$ such that $x, R x$ and $R^{2} x$ are linearly independent. Define the operator $S$ by:

$$
\begin{aligned}
S x & =3 x-R x, \\
S R x & =3 R x-2 R^{2} x, \\
S R^{2} x & =x, \\
S z & =0 \text { for } z \in L,
\end{aligned}
$$

where $L$ is a closed complement of the span of $\left\{x, R x, R^{2} x\right\}$. We have $S \in B(X)$ and $3 \in \sigma(S+R) \cap \sigma(S+2 R)$ but $3 \notin \sigma(S)$. Thus $x, R x$, et $R^{2} x$ are linearly dependent for every $x \in X$. then there exists a quadratic polynomial $p$ such that $p(R)=0$. This polynomial has one of the forms $p(t)=(t-\alpha)(t-\beta)$, or $p(t)=(t-\alpha)^{2}$, or $p(t)=t(t-\alpha)$ or $p(t)=t^{2}$, where $\alpha \neq 0 \neq \beta \neq \alpha$. By the standard decomposition of algebraic operators and since $\operatorname{rank} R>1$, we see that $R$ has a finite dimensional invariant subspace $W$ such that $R / W$ has a matrix representation

$$
\begin{gathered}
\left(\begin{array}{ll}
\alpha & 0 \\
0 & \beta
\end{array}\right), \text { or }\left(\begin{array}{ll}
\alpha & 1 \\
0 & \alpha
\end{array}\right) \text {, or } \\
\left(\begin{array}{lll}
\alpha & 0 & 0 \\
0 & \alpha & 0 \\
0 & 0 & 0
\end{array}\right), \text { or }\left(\begin{array}{ll}
0 & 1 \\
0 & 0
\end{array}\right) \oplus\left(\begin{array}{ll}
0 & 1 \\
0 & 0
\end{array}\right),
\end{gathered}
$$

respectively. we consider a complement $Z$ of $W$ in $X$ and a operator $T$ such that $T / Z=0$ and $T / W$ has matrix representation

$$
\begin{gathered}
\left(\begin{array}{cc}
-3 \alpha / 2 & \beta / 2 \\
\alpha / 2 & -3 \beta / 2
\end{array}\right), \text { or }\left(\begin{array}{cc}
1 & -1 \\
\alpha^{2} & 1
\end{array}\right) \text {, or }\left(\begin{array}{ccc}
0 & 0 & 0 \\
0 & 2 \alpha & 2 \alpha \\
0 & -2 \alpha & -2 \alpha
\end{array}\right) \text {, or } \\
\left(\begin{array}{cc}
0 & 0 \\
1 & 0
\end{array}\right) \oplus\left(\begin{array}{ll}
0 & 0 \\
2 & 0
\end{array}\right),
\end{gathered}
$$

respectively. We have $T \in F(X)$ and there exists $\lambda \in \sigma(T+R) \cap \sigma(T+2 R)$ but $\lambda \notin \sigma(T)$, namely $\lambda=0$ or $\lambda=\alpha+1$ or $\lambda=2 \alpha$ or $\lambda=\sqrt{2}$.

Lemma 2.4. Let $\Phi: B(X) \longrightarrow B(Y)$ be a spectrum preserving additive transformation such that $F(Y) \subset \operatorname{Im} \Phi$ and $R \in B(X)$. Then $\operatorname{rank} \Phi(R)=1$ if and only if rank $R=1$. 
Proof. Let $R \in B(X)$. If $R$ is of rank one, we apply the Lemma 2.3 to have $\sigma(T+R) \cap \sigma(T+2 R) \subset \sigma(T)$, for every $T \in F(X)$. Since $\Phi$ is additive and preserves the spectrum, we have $\sigma(\Phi(T)+\Phi(R)) \cap \sigma(\Phi(T)+2 \Phi(R)) \subset \sigma(\Phi(T))$, for every $T \in F(X)$. By the hypothesis $F(Y) \subset \operatorname{Im} \Phi$ and the lemma 2.2, we have $\sigma(S+\Phi(R)) \cap \sigma(S+2 \Phi(R)) \subset \sigma(S)$, for every $S \in F(Y)$. We apply the Lemma 2.3 we conclude that $\Phi(R)$ is of rank one. To prove the reverse case, we assume that $\Phi(R)$ is of rank one. by the Lemma 2.2, we have $\operatorname{rank} R \leq$ $\operatorname{rank} \Phi(R)$ and therefore $R$ is of rank one.

Lemma 2.5. Let $\Phi: B(X) \longrightarrow B(Y)$ be a spectrum preserving additive transformation such that $F(Y) \subset \operatorname{Im} \Phi$ and $R \in F(X)$. Then

$$
\operatorname{Tr}[\Phi(R) \Phi(T)]=\operatorname{Tr}(R T)
$$

for every $T \in B(X)$.

Proof. Let us fix $T \in B(X)$ and $R \in F(X)$.

If $R$ is a non nilpotent operator of rank one, we have for every $r \in \mathbb{C}^{*}$ that $\rho(T+r R)=|r| \rho\left(\frac{T}{r}+R\right)$. Since $\sigma(R)$ is a finite set, by the Newburyh's Theorem (see [8] Corollary 3.4.5) we have $\rho(T+r R) \rightarrow+\infty$ when $|r| \rightarrow+\infty$, because $\rho\left(\frac{T}{r}+R\right) \rightarrow \rho(R)$ when $|r| \rightarrow+\infty$ and $\rho(R) \neq 0$. It holds that there exists $\alpha>\rho(T)$ such that $|r|>\alpha$ implies $\rho(T+r R)>\rho(T)$. Thus $\sigma(T+r R)$ meets the unbounded component $U$ of of $\mathbb{C}-\sigma(T)$.

The trace of an operator of rank one is equal to non zero element in its spectrum if this element exists or zero in the opposite case. Therefore, $\lambda \in$ $\sigma(T+r R) \cap U$ if and only if $\lambda \in U$ and $\frac{1}{r}=\operatorname{Tr}\left[(\lambda-T)^{-1} R\right]$. Since $\Phi$ preserves the spectrum, we have $\lambda \in \sigma(T+r R) \cap U$ if and only if $\lambda \in \sigma(\Phi(T)+r \Phi(R)) \cap$ $U$, which is equivalent to $\lambda \in U$ and $\frac{1}{r}=\operatorname{Tr}\left[(\lambda-\Phi(T))^{-1} \Phi(R)\right]$, because $\Phi(R)$ is of rank one (Lemma 2.4). The functions $h(\lambda)=\operatorname{Tr}\left[(\lambda-T)^{-1} R\right]$ and $k(\lambda)=\operatorname{Tr}\left[(\lambda-\Phi(T))^{-1} \Phi(R)\right]$ are holomorphics on $U$ and they coincide on $E=\cup_{\left\{r \in Q^{*},|r|>\alpha\right\}}\left\{\lambda \in U ; h(\lambda)=\frac{1}{r}\right\}$. It has been proved by B. Aupetit pp:66[7] that $E$ is not a discrete set, so we apply the identity principle for analytic functions we conclude that $h$ and $k$ are equal on $U$.

For $|\lambda|>\rho(T)=\rho(\Phi(T))$, we develop $h$ and $k$ on series, we have

$$
\operatorname{Tr}\left[\frac{\Phi(R)}{\lambda}+\frac{\Phi(R) \Phi(T)}{\lambda^{2}}+\ldots\right]=\operatorname{Tr}\left[\frac{R}{\lambda}+\frac{R T}{\lambda^{2}}+\ldots\right] .
$$

The fact that $\operatorname{Tr}[\Phi(R)]=\operatorname{Tr}(R)$, we have $\operatorname{Tr}[\Phi(R) \Phi(T)]=\operatorname{Tr}(R T)$.

In the case where $R$ is a nilpotent operator of rank one, it is of the form $R=x \otimes f$ where $f(x)=0$, for some $x \in X$ and $f \in X^{\prime}$. By the HahnBanach Theorem, there exists $g \in X^{\prime}$ such that $g(x)=1$. We have $x \otimes f=$ 
$x \otimes(f+g)-x \otimes g$ and each of the operators $x \otimes(f+g)$ and $x \otimes g$ is a nilpotent operator of rank one. We apply the additivity of $\Phi$ and the argument used in the first case for the operators $x \otimes(f+g)$ and $x \otimes g$ to conclude that $\operatorname{Tr}[\Phi(R) \Phi(T)]=\operatorname{Tr}(R T$.

Every finite rank operator is a finite sum of some operators of rank one ([6] Corollary 2.17 ), then, we have from the above cases and the properties of the trace that $\operatorname{Tr}[\Phi(R) \Phi(T)]=\operatorname{Tr}(R T$.

Lemma 2.6. Let $\Phi: B(X) \longrightarrow B(Y)$ be a spectrum preserving additive transformation such that $F(Y) \subset \operatorname{Im} \Phi$. Then $\Phi$ is $\mathcal{C}$-linear.

Proof. Let $T \in B(X)$ and $\alpha \in \mathbb{C}$. By Lemma 2.5 and the properties of the trace we have

$$
\begin{aligned}
\operatorname{Tr}[(\Phi(\alpha T)-\alpha \Phi(T)) \Phi(R)] & =\operatorname{Tr}[\Phi(\alpha T) \Phi(R)]-\operatorname{Tr}[\alpha \Phi(T) \Phi(R)] \\
& =\operatorname{Tr}[\Phi(\alpha T) \Phi(R)]-\alpha \operatorname{Tr}[\Phi(T) \Phi(R)] \\
& =\operatorname{Tr}(\alpha T R)-\alpha \operatorname{Tr}(\operatorname{TR}) \\
& =0,
\end{aligned}
$$

for every $R \in F(X)$. Since $F(Y) \subset \operatorname{Im} \Phi$, we apply Lemma 2.2 to conclude that $\operatorname{Tr}[(\Phi(\alpha T)-\alpha \Phi(T)) S]=0$ for every $S \in F(Y)$. It holds by Lemma 2.1 that $\Phi(\alpha T)-\alpha \Phi(T)=0$.

Lemma 2.7. Let $\Phi: B(X) \longrightarrow B(Y)$ be a spectrum preserving additive transformation such that $F(Y) \subset \operatorname{Im} \Phi$. Then $\Phi(\lambda)=\lambda$.

Proof. Let $\lambda \in \mathbb{C}$ and $R \in F_{1}(X)$. By Lemma 2.5 and the properties of the trace, we have

$$
\begin{aligned}
\operatorname{Tr}[(\Phi(\lambda)-\lambda) \Phi(R)] & =\operatorname{Tr}[\Phi(\lambda) \Phi(R)]-\operatorname{Tr}(\lambda \Phi(R)) \\
& =\operatorname{Tr}(\lambda R)-\lambda \operatorname{Tr}(\Phi(R)) \\
& =\lambda \operatorname{Tr}(R)-\lambda \operatorname{Tr}(R) \\
& =0 .
\end{aligned}
$$

By the Lemma 2.4 we have $\Phi\left(F_{1}(X)\right)=F_{1}(Y)$ and therefore we have $\operatorname{Tr}[(\Phi(\lambda)-$ $\lambda) S]=0$, for every $S \in F_{1}(Y)$. Since every operator of $F(Y)$ is a finite sum of some operators of rank one, it holds that $\operatorname{Tr}[(\Phi(\lambda)-\lambda) S]=0$ for every $S \in F(Y)$. the Lemma 2.1 implies that $\Phi(\lambda)=\lambda$. 


\section{Proof of Main Results}

Proof of Theorem 2.1. The lemmas 2.2, 2.6 and 2.7 show that $\Phi$ is injective, $\mathbb{C}$-linear and $\Phi(\lambda)=\lambda$ for every $\lambda \in \mathbb{C}$ respectively. Then, we are in the hypotheses which permited to A. R. Sourour in [18] (Theorem 3.4) to prove that either

$$
\Phi(T)=A T A^{-1} \text { for every } T \in B(X),
$$

where $A: X \longrightarrow Y$ is a bijective linear transformation, or

$$
\Phi(T)=B T^{*} B^{-1} \text { for every } T \in B(X),
$$

where $B: X^{\prime} \longrightarrow Y$ is a bijective linear transformation. Now, we prove that $A$ and $B$ are bounded. We start by situation (1), let $x \in X, f \in X^{\prime}, T \in B(X)$ and $\lambda \notin \sigma(T)$. We have, $\lambda \in \sigma(T+x \otimes f)$ if and only if $f\left[(\lambda-T)^{-1} x\right]=$ 1. Since $\Phi$ preserves the spectrum and $\Phi\left(x \otimes f=A x \otimes\left(A^{-1}\right)^{*} f\right.$, it holds $f\left[(\lambda-T)^{-1} x\right]=1$ if and only if $\left(A^{-1}\right)^{*} f\left[\left(\lambda-A T A^{-1}\right)^{-1} A x\right]=1$. we deduce that $f\left[(\lambda-T)^{-1} x=\left(A^{-1}\right)^{*} f\left[\left(\lambda-A T A^{-1}\right)^{-1} A x\right]\right.$, because $A$ and $f$ are linears. By the Closed Graph Theorem, we can easily establish that $A$ is bounded, and by the same argument, we can prove that $B$ is also bounded.

Proof of Corollary 2.1. The Theorem 2.1 shows that either $\Phi(T)=A T A^{-1}$, or $\Phi(T)=B T^{*} B^{-1}$ for every $T \in B(X)$, where $A: X \longrightarrow X$ and $B: X^{\prime} \longrightarrow X$ are bijective bounded linear transformations. In the case where $\Phi(T)=A T A^{-1}$, it is easy to show that $\Phi$ is surjective. In the other case, let $K: X \longrightarrow X^{\prime \prime}$ be the natural embedding of $X$ into $X^{\prime \prime}$. We have $\Phi(x \otimes f)=B f \otimes\left(B^{-1}\right)^{*} K x$, it holds that $\left(B^{-1}\right)^{*} K$ is bijective, which implies that $K$ is bijective. Then $X$ is reflexive. Now it is easy to establish that in this case $\Phi$ is surjective.

\section{References}

[1] M. Ech-chérif El Kettani and E.El Bouchibti, Sur les transformations additives qui consevent le spectre ponctuel, Extracta Mathematicae, 16, No. 3 (2001), 343-349.

[2] Mustapha Ech-chérif El Kettani and EL Houcine El Bouchibti, Sur les transformations additives qui conservent le spectre de surjection, Extracta Mathematicae, 18, No 1 (2003), 57-64.

[3] Mustapha Ech-chérif El Kettani and EL Houcine El Bouchibti, On automatic surjectivity of some additive transformations, Proyecciones, 23, No. 2 (2004), 111-121.

[4] Mustapha Ech-chérif El Kettan and Hassane Benbouziane, Surjective maps preserving local spectral radius, Internationan Mathematical Forum, 9, No. 11 (2014), 515-522.

[5] B. Aupetit, Une généralisation du théorème de Gleason-Kahane-Zelazko pour les algèbres de Banach, Pacific. J. Math., 85 (1979), 11-17. 
[6] B. Aupetit, H. du Toit Mouton, Trace and determinant in Banach algebras, Studia Math., 121 (1996), 115-136.

[7] B. Aupetit, Sur les transformations qui conservent le spectre, Banach Algebras, 97, De Gryter, Berlin (1998), 55-78.

[8] B. Aupetit, A Primer on Spectral Theory, Springer, New-York, 1991.

[9] M. Brešar and P. Šemrl, Linear maps preserving the spectral radius, J. Funct. Anal., 142 (1996), 360-168.

[10] Fillmore, Sums of operators with square-zero, Acta. Sci. Math. Szeged., 28 (1967), 285288.

[11] A.A. Jafarian and A.R. Sourour, Spectrum preserving linear maps, J. Funct. Anal., 66 (1986), 255-261.

[12] M. Omladič, P. Š emrl, Spectrum preserving additive maps, Linear. Algebras Appl., 153 (1991), 67-72.

[13] M. Omladič, P. Šemrl, Additive mapping preserving operators of rank one, Linear. Algebra. App., 182 (1993), 239-256.

[14] W. Rudin, Functional Analysis, McGraw-Hill, Second Edition, 1991.

[15] P. Šemrl, Spectrally bounded linear maps on B(H), Quat. J. Math., Oxford, 49 (1998), 87-92.

[16] P. Šemrl, Linear maps that preserve the nilpotent operators, Acta. Sci. Math., 61 (1995), 523-534.

[17] S. Sakai, $C^{*}$-Algebras and $W^{*}$-Algebras, Springer, New-York, 1971.

[18] A.R. Sourour, Invertibility preserving linear maps on $\mathcal{L}(X)$, Trans. Amer. Soc., 348 (1996), 13-30. 